\title{
Factors influencing and changes in childhood vaccination coverage over time in Bangladesh: $A$ multilevel mixed-effects analysis
}

\section{Satyajit Kundu ( $\nabla$ satyajitnfs@gmail.com )}

Department of Biochemistry and Food Analysis, Patuakhali Science and Technology University, Patuakhali - 8602, Bangladesh https://orcid.org/0000-0001-9610-1479

\section{Subarna Kundu}

Statistics Discipline, Khulna University, Khulna, Bangladesh

\section{Abdul-Aziz Seidu}

College of Public Health, Medical and Veterinary Services, James Cook University, Australia

\section{Joshua Okyere}

Department of Population and Health, University of Cape Coast, Cape Coast, Ghana.

\section{Susmita Ghosh}

Department of Food Technology and Nutrition Science, Noakhali Science and Technology University, Noakhali, Bangladesh

\section{Ahmed Hossain}

Department of Public Health, North South University, Dhaka, Bangladesh.

\section{Najim Z. Alshahrani}

Department of Family and Community Medicine, Faculty of Medicine, University of Jeddah, Jeddah 21589, Saudi Arabia

\section{Md. Hasan Al Banna}

Department of Food Microbiology, Patuakhali Science and Technology University, Patuakhali- 8602, Bangladesh

\section{Md. Ashfikur Rahman}

Development Studies Discipline, Khulna University, Khulna 9208, Bangladesh

\section{Bright Opoku Ahinkorah}

School of Public Health, Faculty of Health, University of Technology Sydney, Sydney, Australia.

\section{Research Article}

Keywords: Childhood vaccination, Immunization, Prevalence, BDHS, Bangladesh

Posted Date: January 3rd, 2022

DOl: https://doi.org/10.21203/rs.3.rs-722674/v2 
License: (c) (i) This work is licensed under a Creative Commons Attribution 4.0 International License. Read Full License 


\section{Title page}

Title: Factors influencing and changes in childhood vaccination coverage over time in Bangladesh: A multilevel mixed-effects analysis

Authors: Satyajit Kundu1,\#, *, Subarna Kundu', \#, Abdul-Aziz Seidu ${ }^{3,4}$, Joshua Okyere ${ }^{3}$, Susmita Ghosh $^{5,6}$, Ahmed Hossain ${ }^{7}$, Najim Z. Alshahrani ${ }^{8}$, Md. Hasan Al Banna ${ }^{9}$, Md. Ashfikur Rahman ${ }^{10}$ \& Bright Opoku Ahinkorah ${ }^{11}$

\section{Affiliations:}

${ }^{1}$ Department of Biochemistry and Food Analysis, Patuakhali Science and Technology University, Patuakhali 8602, Bangladesh.

${ }^{2}$ Statistics Discipline, Khulna University, Khulna 9208, Bangladesh.

${ }^{3}$ Department of Population and Health, University of Cape Coast, Cape Coast, Ghana.

${ }^{4}$ College of Public Health, Medical and Veterinary Services, James Cook University, Australia.

${ }^{5}$ Department of Food Technology and Nutrition Science, Noakhali Science and Technology University, Noakhali, Bangladesh

${ }^{6}$ Department of Nutrition Science, Purdue University, IN 47907, USA.

${ }^{7}$ Department of Public Health, North South University, Dhaka 1229, Bangladesh.

${ }^{8}$ Department of Family and Community Medicine, Faculty of Medicine, University of Jeddah, Jeddah 21589, Saudi Arabia.

${ }^{9}$ Department of Food Microbiology, Patuakhali Science and Technology University, Patuakhali 8602, Bangladesh.

${ }^{10}$ Development Studies Discipline, Khulna University, Khulna 9208, Bangladesh.

11 School of Public Health, Faculty of Health, University of Technology Sydney, Sydney, Australia.

\# These authors are joint first author for this work.

\section{*Corresponding author:}

\section{Satyajit Kundu}

Department of Biochemistry and Food Analysis, 
Patuakhali Science and Technology University, Patuakhali - 8602, Bangladesh.

Email: satyajitnfs@gmail.com

ORCID: https://orcid.org/0000-0001-9610-1479

\begin{abstract}
There is a dearth in the information of childhood vaccination coverage in Bangladesh. Thus, this study aimed to investigate the associated factors and changes in childhood vaccination coverage over time in Bangladesh. Bangladesh's Demographic and Health Surveys from 2011, 2014, and 2017-18 provided data on vaccination coverage for children aged 12 to 35 months. For three survey periods, multilevel binary logistic regression models were employed. The overall prevalence (weighted) of full vaccination among children aged 12-35 months were $86.17 \%$ in 2011 and $85.13 \%$ in 2014 , and $89.23 \%$ in $2017-18$. Children from families with high wealth index, mothers with higher education, and over the age of 24 and who sought at least four ANC visits, as well as children from urban areas were more likely to receive full vaccination. Rangpur division had the highest change rate of immunization coverage from 2011 to 2014 (2.26\%), whereas Sylhet division had the highest change rate from 2014 to 2017-18 (34.34\%). To improve immunization coverage for Bangladeshi children, policymakers must integrate vaccine programs, paying special attention to mothers without at least a high school education and families with low wealth index. Increased antenatal care visits may also aid in increasing the immunization coverage of their children.
\end{abstract}

Keywords: Childhood vaccination; Immunization; Prevalence; BDHS; Bangladesh

\title{
Introduction
}

Vaccinations are widely acknowledged as one of the safest and most cost-effective ways to protect children against infectious diseases such as tuberculosis and measles ${ }^{1}$. Thus, childhood vaccination has been increasing over the past decades $^{2}$. For example, the vaccination of children against 
diphtheria-tetanus-pertussis (DTP3) increased astoundingly from a global coverage of $20 \%$ in 1980 to $85 \%$ in $2019^{3}$. More profound is the evidence that vaccination averts between 2-3 million deaths attributable to vaccine-preventive diseases such as Diphtheria, Pertussis, Tetanus, and Measles among children under-five every year ${ }^{4,5}$.

Although the world has seen remarkable improvements in childhood vaccination, achieving complete coverage over time remains an important public health concern ${ }^{6}$. Not every child is getting vaccinated. For instance, 19.4 million infants did not receive basic vaccination as at the end of $2019^{7,8}$. Most of these deficiencies in childhood vaccination coverage are recorded in lowand-middle-income countries (LMICs). The WHO asserts that, in remote rural areas of LMICs, only 1 out of 20 children have access to vaccination ${ }^{9}$. The consequences of not achieving complete childhood vaccination cannot be underestimated. Vaccination provides an opportunity to avert millions of deaths and a host of vaccine-preventable diseases among children ${ }^{1}$. Within the framework of the WHO, children who miss scheduled vaccinations for any reason due to health facility problems such as canceled vaccination schedules or vaccine stock-outs are categorized as having incomplete vaccination ${ }^{10}$. Denying children access to a complete dose of vaccines would be catastrophic as a countless number of children will die or develop some form of disabilities ${ }^{11}$. As such, it is imperative to understand the nuances that characterize childhood vaccination coverage over time.

Available evidence suggests that there are several factors that influence the uptake of vaccination for children under-five. For instance, a qualitative study by Jalloh et al. ${ }^{12}$ indicates that perceived beliefs about the side effects coupled with concerns about receiving multiple vaccines on the same day were significant barriers to the uptake of childhood vaccination and its coverage. Also, other 
studies from Ghana ${ }^{13}$ and Nigeria ${ }^{14}$ suggest that urban residency and higher maternal education are associated with higher likelihoods of complete childhood vaccination coverage.

Since 1979, the Government of Bangladesh has started vaccinations against six preventable diseases (tuberculosis; diphtheria, pertussis, and tetanus; polio; and measles) through the Expanded Program on Immunization (EPI $)^{15}$. According to the Bangladesh Immunization guidelines, children who have received one dose of the vaccine against tuberculosis, Bacille Calmette-Guerin (BCG), three doses of a pentavalent vaccine (DPT, Hib, and HepB), three doses of the polio vaccine (excluding the polio vaccine given at birth), and one dose of the measles and rubella vaccine are considered as fully vaccinated, if they would miss any of the recommended doses they will be considered as partially vaccinated ${ }^{15,16}$.

Bangladesh as a country has attained significant heights in reducing childhood mortality; this is seen in the country's capacity to meet the Millennium Development Goal $4{ }^{17}$. Through the implementation of the WHO's Expanded Programme on Immunization (EPI), Bangladesh was able to commit sufficiently towards the promotion of childhood vaccination coverage which saw a sustained impact on childhood mortality, reducing it from 133 deaths per 1000 live births in 1993 to 46 deaths per 1000 in $2014^{17}$. In a bid to augment efforts toward childhood vaccination coverage, the Bangladeshi government came up with three nationwide supplementary immunization activities (SIAs) from 2000 to $2016^{18}$. These initiatives resulted in a significant decline in the incidence of measles, from 14,745 incident cases in 2010 to 972 in $2016^{12,18}$. Nevertheless, Bangladesh faces high levels of childhood mortality which places the country among the top ten countries with the highest rates of childhood mortality, with vaccine-preventable diseases being the causes of these mortalities ${ }^{12,19}$. This makes Bangladesh an opportune context to understand childhood vaccine coverage and its concomitant factors. 
Bangladesh relies on composite estimates based on administrative coverage data gathered from healthcare providers, population-based household surveys, and governmental agencies ${ }^{17}$. However, due to the incompleteness and mistakes associated with the original collection of data on childhood immunization in Bangladesh, such estimates are frequently incorrect ${ }^{17}$. As a result, utilizing a nationally representative survey provides much more clarity and strong data to investigate the factors that influence vaccination coverage ${ }^{20}$. Using nationally representative data from the BDHS 2011, 2014, and 2017-18, the study aims to track the vaccination status of children aged 12 to 35 months and examine the factors that influence full immunization coverage in Bangladesh.

\section{Methods}

Data source and study design. The current study utilized three recent nationally representative cross-sectional Bangladesh demographic health survey data (BDHS 2011, 2017, and 2017-18). The survey included both urban and rural households from all administrative regions of Bangladesh. The data was collected using two-stage stratified cluster sampling design of the household. At the first stage, enumeration areas (EAs) were selected with probability proportional to sizes like 672 in 2017-18, 600 in both 2014 and 2011 BDHS respectively. After getting the EAs (cluster), on average 30 households were selected from each cluster using systematic sample selection. Detailed information on the sampling design could be found in the BDHS survey reports $^{16,21,22}$.

Outcome measure. Vaccination status among children aged 12-35 months was assessed and the age range was also used in the several previous studies ${ }^{23-26}$. Based on the Expanded Program on Immunization (EPI) of the government of Bangladesh, the basic vaccines for children are: Bacille Calmette-Guérin (BCG vaccine); diphtheria, pertussis, and tetanus (DPT vaccine); poliomyelitis 
(oral polio [OPV] vaccine); and measles (measles vaccine) ${ }^{21}$. Children aged 12-35 months were considered to be fully vaccinated if they got the BCG vaccine at birth, three doses of polio, three doses of DPT and one dose of measles at any time before the survey (Figure 1). Partially vaccinated were defined as lacking any dose of the basic vaccination. While those who failed to take the recommended doses of vaccine were categorized as "none". Vaccination coverage information was collected in two ways from the vaccination card or from the mother's verbal report. For final analysis, vaccination status was dichotomized as "fully vaccinated" and "not fully vaccinated" (merging partially vaccinated and no vaccinated). Hepatitis B vaccine (1-3 dose), Haemophilus influenzae type B vaccine, and inactivated polio vaccine (IPV) were not included in the current study since information on these were not available in 2011 and 2014 BDHS.

\section{Insert Figure 1}

Figure 1. Basic vaccination administration schedule for children under 12 months in Bangladesh.

Explanatory variables. According to the guidance of reviewed literature and the availability of the variables, several demographic and health variables were included in this current analysis $^{1,27,28}$. The included variables for this study are child age, mother's age, mother's education, antenatal care (ANC) visit, place of residence, division, gender, place of delivery, number of children. Children aged 12-35 months were selected to conduct the study who were categorized as "12-23 months", "24-35 months". Maternal age was categorized as "less than 24 years", "24-34 years", and "above 34 years"29,30. The household wealth index was calculated using principal component analysis of the different household assets ${ }^{22}$. The wealth index of the household was recategorized as "poor" (poorest or poorer), "middle", and "rich" (richer, richest). Media access was measured by asking mothers about the number of times they read a newspaper, 
listen to the radio, and watch television. Adding these variables media access was recategorized as "less than once a week" or "at least once a week". Place of delivery was recategorized as "home", "health facility delivery" (public and private health care facility), and "others" (don't know, didn't response, and missing). Antenatal care visits of mothers were categorized into "no visit", "1-3 visits", "4 or more visits", and "others". Maternal education was classified into "no education", "primary", "secondary" and "higher" education. Currently, Bangladesh has eight administrative divisions including Barisal, Dhaka, Chittagong, Khulna, Mymensingh, Rajshahi, Rangpur and Sylhet. However, Mymensingh division was separated from Dhaka division in $2015^{31}$. That's why information of Mymensingh division was not available separately in 2011 and 2014 BDHS survey, and we merged the Mymensingh and Dhaka divisions into "Dhaka" in order to make the analysis consistent.

Statistical analysis. In this study, we analysed data from across the three different surveys and pooled data from the three surveys as well to understand the overall vaccination coverage as well as the changes in vaccination coverage across surveys. We used descriptive statistics to show the characteristics of respondents and the differences in the vaccination coverage between categories were tested using Pearson chi-square analysis. To explore the weighted prevalence of fully vaccination of children across different sub-categories, we used the "svy" command for assigning the sample weight to adjust for clustering effect and sample stratification. Additionally, maps showing the spatial distribution of change rates of full vaccination coverage in the three surveys were created. The change rates of full vaccination coverage over time within each division were calculated using the formula:

$$
\frac{((\% \text { fully vaccinated in the recent year })-(\% \text { fully vaccinated in the previous year }))}{(\% \text { fully vaccinated in the previous year })} \times 100
$$


Considering the two-stage stratified cluster sampling of BDHSs, we used multilevel (2-level) logistic regression (MLLR) analysis to identify the factors influencing full vaccination coverage by reducing the cluster effects. Because a single-level analysis would not be appropriate for analyzing such data sets that have hierarchical structures ${ }^{32}$. We considered the enumeration areas (clusters) as level-2 factor for all the regression models. Both the chi-square and MLLR analysis were executed for each survey year separately as well as for pooled data. Multicollinearity among independent variables was checked using variance inflation factor (VIF). After employing the multilevel models, the intra-class correlation coefficient (ICC) was also estimated to check the cluster effects on the outcome variable. The adjusted odds ratio (AOR) along with $95 \%$ confidence interval $(\mathrm{CI})$ were used to interpret the findings and 5\% significance level was considered. All analyses were performed using the statistical package SPSS (version 23.0) and STATA (version 17.0). Spatial analysis along with maps were generated using ArcGIS (version 10.8).

Ethical approval. This study used a secondary data analysis of publicly available survey data from the MEASURE DHS program (https://www.dhsprogram.com). These DHS survey reports are publicly available and the datasets are accessible upon application. We requested the data set, and permission was granted to download and use the data for this study. Thus, further ethical approval for this study was not required. All the procedures were performed in accordance with the relevant guidelines and regulations.

\section{Results}

The current study estimated the vaccination coverage among 12-35 months aged children in Bangladesh over three time periods from the data of BDHS (2011, 2014, and 2017-18). Though in 2014 the coverage of full vaccination (85.13\%) is slightly lower than the previous BDHS 2011 (86.17\%), the status of full vaccination has increased significantly over time (89.23\% in 2017-18) 
$(\mathrm{p}<0.001)$. The vaccination coverage which was reported either from vaccination cards or by mother recall has been also significantly increased over time. In 2011, the coverage of BCG was $97.33 \%$, in 2014 it was $97.70 \%$ and while in $2017-18$ the coverage increased to $98.50 \%$. All three

doses of polio (Polio 1, Polio2, Polio 3) vaccines were observed to be increased over time and a significant increment $(p<0.001)$ was seen in the full coverage of OPV (1-3) though a little decrease has been observed in 2014 DHS. Similarly, the full coverage of DTP was also increased from 2011 (92.96\%) to 2017-18 $(96.01 \%)(\mathrm{p}<0.001)$ (Table 1).

\section{Insert Table 1}

Table 1. Vaccination coverage estimates (weighted percentage) for children 12-35 months of age by survey year

The overall prevalence (weighted) of full vaccination coverage was $86.71 \%$ in $2011,85.13 \%$ in 2014, and $89.23 \%$ in $2017-18$ where the pooled prevalence was $87.06 \%$. The pooled analysis shows that all the variables except sex of children and maternal age were found to be significantly associated with the full vaccination coverage (all $\mathrm{p}<0.05$ ). The pooled data also shows that the highest percentage (weighted) of full vaccination was observed among children from Rangpur division (92.28\%) followed by Khulna division $(90.55 \%)$ while the worst situation was found in Sylhet division (Table 2).

Figure 2 depicts the geographical pattern of the change rate in full vaccination coverage throughout three survey periods. While most divisions saw a reduction in full vaccination coverage over time from 2011 to 2014, the highest positive change rate from 2011 to 2014 was found in Rangpur division (2.26\%) and Sylhet division had the worst scenario (-21.24\%). Interestingly, from 2014 to 2017-18, all divisions experienced an increase in vaccination status except Rangpur 
division $(-1.25 \%)$, while the highest improvement regarding the change rate was found in Sylhet division $(34.34 \%)$.

\section{Insert Figure 2}

Figure 2. Spatial distribution of change rate in vaccination status among children aged 12-35 months old from BDHS 2011 to 2014 and BDHS 2014 to 2017-18.

\section{Insert Table 2}

Table 2. Bivariate distribution of basic vaccination coverage (full) by socio-demographic variables among children aged 12-35 months in Bangladesh

Random-effect parameters of adjusted regression models suggest that clustering variations are present in the outcome measure among enumerations (clusters). Considering the stratified (twostage) sampling design of the survey, two-level logistic regression analysis approach was employed that allows to remove the clustering effect to ensure precise findings. All variables were included in the adjusted model for controlling the confounding effect of the covariates. The adjusted regression model of pooled analysis demonstrates that age of the child, maternal age and education, ANC visit of the mother, household wealth index, place of residence and community literacy level of women were significantly associated with the coverage of the full vaccination.

We found that children of mothers having secondary, and higher education experienced a better exposure to full vaccination than the mothers who had no formal education in all three waves of BDHS and pooled analysis. Pooled analysis found that children from households with rich wealth index were $27 \%(\mathrm{AOR}=1.27,95 \% \mathrm{CI}: 1.04$ to 1.55$)$ more likely to get full vaccination compared to those from families with poor wealth index. From the pooled analysis, we also found that if the mother sought at least 4 ANC visits then the likelihood of getting full vaccination was increased by $77 \%(\mathrm{AOR}=1.77,95 \% \mathrm{CI}: 1.44$ to 2.17$)$ compared to those having no ANC visit. The coverage 
of full vaccination was higher among the children with mothers aged 25-34 years and above 34 years compared to children of mothers aged $<25$ years in 2014 and 2017-18 and in pooled analysis. Children from urban areas were more likely to get fully vaccinated than their rural counterparts and the association is found to be significant in 2014 ( $\mathrm{AOR}=1.48,95 \% \mathrm{CI}: 1.06$ to 2.06 ) and in pooled analysis ( $\mathrm{AOR}=1.25,95 \% \mathrm{CI}: 1.05$ to 1.49 ). This study also shows that higher community literacy level of women was associated with the higher odds of getting full vaccination in 2011 ( $\mathrm{AOR}=1.46,95 \% \mathrm{CI}: 1.04$ to 2.06$), 2017-18(\mathrm{AOR}=1.46,95 \% \mathrm{CI}: 1.03$ to 2.05$)$, and in pooled analysis (AOR=1.36, 95\% CI: 1.12 to 1.65 ). Significant regional variation was also observed in pooled analysis. Children from Rangpur division were more likely to get full vaccination compared to Barisal division (AOR=1.72.95\% CI: 1.32 to 2.79 ) (Table 3).

\section{Insert Table 3}

Table 3. Multilevel regression analysis showing the factors associated with full vaccination coverage of children aged 12-35 months in Bangladesh

\section{Discussion}

The Sustainable Development Goal encourages countries and governments to take steps to ensure that their national immunization programs are fully vaccinated by $2030^{33}$. As a result, understanding the factors that determine vaccination coverage over time is crucial for Bangladesh to assess its progress toward universal childhood immunization. The goal was to track the vaccination status of children aged 12 to 35 months and look at the factors that influence full immunization coverage. Overall, our findings show that the percentage of those who have had complete vaccination has risen significantly over time. This is substantiated by a recent Bangladeshi study that demonstrated a slight increase in complete immunization coverage over time ${ }^{17}$. This increment in full vaccination coverage may possibly be attributable to conscious 
health policies such as the three nationwide supplementary immunization activities (SIAs) from 2000 to 2016 which were rolled out by the Bangladeshi government ${ }^{18}$. Despite the fact that full vaccination status for all vaccines increased significantly over time, BCG continuously had the greatest full vaccination coverage. This is consistent with the findings of Boulton et al., who found that BCG had the highest full vaccination coverage compared to the other vaccinations ${ }^{17}$. This observation could be explained by the fact that, unlike other vaccines that are given after a few weeks (such as OPV and DTP) or months (such as measles), BCG is given at birth, minimizing the risk of not getting immunized ${ }^{34}$.

We also observed some divisional variations from our spatial analysis of the distribution of change rate in relation to childhood vaccination coverage. It can be observed from our study that, between 2011 to 2014, a positive change rate was observed in Rangpur division while the worst situation was found in Sylhet division. This is in agreement with the findings of Sheikh et al. ${ }^{15}$. This higher likelihood of incomplete vaccination in the Sylhet division was linked to the remote hilly and riverine nature of the area coupled with the fragile communication system of this area ${ }^{15}$. However, between 2014 to 2017-18, all divisions experienced an increase in vaccination status except Rangpur division, with the highest improvement in change rate being recorded in the Sylhet division.

Our findings also reveal that the factors associated with childhood vaccination coverage over time spread across child, maternal and contextual factors. Concerning the child factors, our analyses revealed that the current age of the child was significantly associated with full vaccination coverage. Thus, the likelihood of full vaccination increased with increased age in children. Hence, older children were more likely to be fully vaccinated. The findings align with evidence from DR Congo $^{1}$. First, childhood vaccinations are scheduled and for that matter, it is expected that older 
children will be at a higher likelihood of being fully vaccinated. Moreover, Bangladesh has had a fair share of mass vaccination programs over the years ${ }^{35}$. Such mass vaccinations, in the perspective of Alfonso et al. ${ }^{1}$, lead to catch-up vaccination with age, thereby predisposing older children (24-35 months) to a higher possibility of being fully vaccinated compared to those aged 12-23 months.

In relation to maternal factors and childhood vaccination coverage, our findings revealed that maternal age and education were significantly associated with vaccination coverage over time. This result is substantiated by earlier studies conducted in Bangladesh ${ }^{36,37}$, DR Congo ${ }^{38}$, and Ethiopia $^{4}$. We found that children born to mothers older than 34 years were more likely to be fully vaccinated as compared to those born to mothers younger than age 24 years. Often, mothers younger than age 24 are inexperienced and therefore may not be knowledgeable about the timing and relevance of ensuring that their children receive the full dose of vaccinations. Hence, explaining the lower odds of full vaccination coverage among children born to mothers younger than age 24. Children born to mothers who had formal education had higher likelihoods of full vaccination coverage. A plausible justification of the effect of maternal education on childhood vaccination coverage could be that mothers with formal education are more likely to be knowledgeable about childhood vaccination, its benefits, as well as being aware of the schedules ${ }^{39}$.

At the contextual level, urban residence, and attending at least 4 ANC visits were significantly associated with increased full vaccination coverage. This finding is consistent with studies from Bangladesh $^{1}$, as well as studies from Ethiopia ${ }^{39}$ and Ghana ${ }^{40}$. Attending at least 4 ANC visits provide mothers with the opportunity to be exposed to more health education and sensitization messages about the importance of ensuring full vaccination of their children, as well as providing parents with vaccination schedules ${ }^{39}$. Another possible explanation for the findings is that mothers 
who receive more ANC visits may gain satisfaction with healthcare access which could potentially translate into higher vaccination coverage ${ }^{41}$. Concerning the urbanicity of our findings, it could be argued from the perspective that urban areas have a substantial proportion of health professionals and health facilities; therefore, more children in the urban areas are likely to have access to full vaccination coverage compared to their counterparts in the rural areas ${ }^{13,42}$.

Consistent with a related study in Bangladesh ${ }^{15}$, the present study revealed that children born to rich wealth indexed households were more likely to receive full vaccination as compared to those born into poor wealth indexed households. Usually, mothers from poor households experience high level of food insecurity and deprivation which tends to make vaccination a low priority for such mothers ${ }^{15,43}$. Thus, explaining the observed association in this study. The study also revealed that higher community literacy level of women was associated with the higher odds of getting full vaccination. This observation can be attributed to the fact that vaccination is influenced by community and societal norms ${ }^{44}$. As a result, increased community literacy means that the community has been exposed to the need and necessity for vaccination on a large scale, making them more inclined to embrace vaccination practices and encourage full vaccination of children.

Our study has several strengths. The dataset used in this study was nationwide in nature, allowing the findings to be extended to children all over Bangladesh. Furthermore, the DHS dataset is a thoroughly tested, repeatable, standardized, and comprehensive survey. Examining variations in vaccination coverage across surveys and comparing them to an analysis of the aggregated data from all the surveys provides a holistic picture of the country's vaccine coverage. This, together with the application of stringent statistical processes, ensures the validity of our findings and the study's replicability in other settings. Nonetheless, there are some inherent limitations to the study that should be considered when interpreting our findings. Because the BDHS uses a cross-sectional 
design, causality cannot be proven. Furthermore, information on the status of child vaccination is based on either immunization cards or women's self-reports; hence, recall bias may exist, resulting in an under- or overestimation of vaccination coverage.

\section{Conclusion}

According to the study, children's immunization coverage has risen over time. Childhood vaccine interventions should focus on mothers with no formal education, younger mothers (less than 24 years), and children born to mothers in impoverished families to close the gap in full childhood immunization in Bangladesh. In order to attain full childhood immunization, the government must also promote ANC attendance and community literacy.

\section{Data availability}

The datasets of BDHS 2011, 2014 and 2017-18 are available upon request in the following website: http://dhsprogram.com/data/available-datasets.cfm.

\section{References}

1. Alfonso, V. H. et al. Changes in childhood vaccination coverage over time in the Democratic Republic of the Congo. PLoS One 14, e0217426 (2019).

2. Kim, Y. E. Determinants of childhood vaccination in Nagaland, India: a cross-sectional study with multilevel modelling. BMJ Open 11, e045070 (2021).

3. WHO. Global and regional immunization profile. (2020).

4. Fenta, S. M. \& Fenta, H. M. Individual and community-level determinants of childhood vaccination in Ethiopia. Arch. Public Heal. 79, 1-11 (2021).

5. Zenbaba, D. et al. Determinants of Incomplete Vaccination Among Children Aged 12 to 23 
Months in Gindhir District, Southeastern Ethiopia: Unmatched Case-Control Study. Risk Manag. Healthc. Policy 14, 1669 (2021).

6. Casula, M. \& Toth, F. The 2017 Italian reform on mandatory childhood vaccinations: Analysis of the policy process and early implementation. Health Policy (New. York). 125, 7-11 (2021).

7. Li, X. et al. Estimating the health impact of vaccination against ten pathogens in 98 lowincome and middle-income countries from 2000 to 2030: a modelling study. Lancet 397, 398-408 (2021).

8. Nour, T. Y. et al. Predictors of immunization coverage among 12-23 month old children in Ethiopia: systematic review and meta-analysis. BMC Public Health 20, 1-19 (2020).

9. WHO. Improving influenza vaccine virus selection: Report of the 4th WHO informal consultation, Hong Kong SAR, China, 18-20 November 2015. (World Health Organization, 2015).

10. VanderEnde, K., Gacic-Dobo, M., Diallo, M. S., Conklin, L. M. \& Wallace, A. S. Global routine vaccination coverage-2017. Morb. Mortal. Wkly. Rep. 67, 1261 (2018).

11. WHO. Early childhood development and disability: A discussion paper. (2012).

12. Jalloh, M. F. et al. Rapid behavioral assessment of barriers and opportunities to improve vaccination coverage among displaced Rohingyas in Bangladesh, January 2018. Vaccine 37, 833-838 (2019).

13. Budu, E., Darteh, E. K. M., Ahinkorah, B. O., Seidu, A.-A. \& Dickson, K. S. Trend and determinants of complete vaccination coverage among children aged 12-23 months in Ghana: Analysis of data from the 1998 to 2014 Ghana Demographic and Health Surveys. PLoS One 15, e0239754 (2020). 
14. Ushie, B. A., Fayehun, O. A. \& Ugal, D. B. Trends and patterns of under-5 vaccination in N igeria, 1990-2008: what manner of progress? Child. Care. Health Dev. 40, 267-274 (2014).

15. Sheikh, N. et al. Coverage, timelines, and determinants of incomplete immunization in Bangladesh. Trop. Med. Infect. Dis. 3, 72 (2018).

16. NIPORT, Mitra and Associates, and I. I. Bangladesh Demographic and Health Survey 2014. Dhaka, Bangladesh: NIPORT, Mitra and Associates, and ICF International. (2016).

17. Boulton, M. L., Carlson, B. F., Power, L. E. \& Wagner, A. L. Socioeconomic factors associated with full childhood vaccination in Bangladesh, 2014. Int. J. Infect. Dis. 69, 3540 (2018).

18. Khanal, S. et al. Progress toward measles elimination-Bangladesh, 2000-2016. MMWR. Morb. Mortal. Wkly. Rep. 66, 753 (2017).

19. Unicef. Committing to child survival: a promise renewed. (eSocialSciences, 2015).

20. Cutts, F. T., Claquin, P., Danovaro-Holliday, M. C. \& Rhoda, D. A. Monitoring vaccination coverage: defining the role of surveys. Vaccine 34, 4103-4109 (2016).

21. NIPORT, Ministry of Health and Family Welfare, and I. Bangladesh Demographic and Health Survey 2017-18. Dhaka, Bangladesh: NIPORT/ICF. (2020).

22. NIPORT, Mitra and Associates, and I. I. Bangladesh Demographic and Health Survey 2011. Dhaka, Bangladesh: NIPORT, Mitra and Associates, and ICF International. (2013).

23. Janusz, C. B. et al. Vaccine Delay and Its Association With Undervaccination in Children in Sub-Saharan Africa. Am. J. Prev. Med. 60, S53-S64 (2021).

24. Xeuatvongsa, A., Hachiya, M., Miyano, S., Mizoue, T. \& Kitamura, T. Determination of factors affecting the vaccination status of children aged 12-35 months in Lao People's 
Democratic Republic. Heliyon 3, e00265 (2017).

25. Shrivastwa, N., Gillespie, B. W., Kolenic, G. E., Lepkowski, J. M. \& Boulton, M. L. Predictors of vaccination in India for children aged 12-36 months. Am. J. Prev. Med. 49, S435-S444 (2015).

26. Ichimura, Y. et al. Affecting factors on vaccination status among children aged between 12 and 35 months: Nationwide population-based cross-sectional study in Lao PDR, 2019. Int. J. Infect. Dis. 101, 519 (2020).

27. Odusanya, O. O., Alufohai, E. F., Meurice, F. P. \& Ahonkhai, V. I. Determinants of vaccination coverage in rural Nigeria. BMC Public Health 8, 1-8 (2008).

28. Moran, E. B. et al. Socioeconomic characteristics associated with the introduction of new vaccines and full childhood vaccination in Ghana, 2014. Vaccine 38, 2937-2942 (2020).

29. Khan, J., Shil, A. \& Mohanty, S. K. Hepatitis B vaccination coverage across India: exploring the spatial heterogeneity and contextual determinants. BMC Public Health 19, 114 (2019).

30. Obanewa, O. A. \& Newell, M. L. The role of place of residency in childhood immunisation coverage in Nigeria: Analysis of data from three DHS rounds 2003-2013. BMC Public Health 20, 123 (2020).

31. Wikipedia. Mymensingh Division. (2021). Available at: https://en.wikipedia.org/wiki/Mymensingh_Division. (Accessed: 15th June 2021)

32. Khan, H. R. \& Shaw, E. Multilevel logistic regression analysis applied to binary contraceptive prevalence data. J. Data Sci. 9, 93-110 (2011).

33. Ratzan, S. C. et al. The Salzburg statement on vaccination acceptance. J. Health Commun. 24, 581-583 (2019). 
34. Herrera, O. R., Thornton, T. A., Helms, R. A. \& Foster, S. L. MMR Vaccine: When Is the Right Time for the Second Dose? J. Pediatr. Pharmacol. Ther. 20, 144-148 (2015).

35. Nurunnabi, A. S. M., Mozaffor, M., Hossain, M. A. \& Sony, S. A. Mass Vaccination Programme: Public Health Success and Ethical Issues-Bangladesh Perspective. Bangladesh J. Bioeth. 9, 11-15 (2018).

36. Haque, S. M. R. \& Bari, W. Positive role of maternal education on measles vaccination coverage in Bangladesh. Int J Psychol Behav Sci 3, 11-17 (2013).

37. Rahman, M. \& Obaida-Nasrin, S. Factors affecting acceptance of complete immunization coverage of children under five years in rural Bangladesh. Salud Publica Mex. 52, 134-140 (2010).

38. Acharya, P., Kismul, H., Mapatano, M. A. \& Hatløy, A. Individual-and community-level determinants of child immunization in the Democratic Republic of Congo: a multilevel analysis. PLoS One 13, e0202742 (2018).

39. Kinfe, Y., Gebre, H. \& Bekele, A. Factors associated with full immunization of children 12-23 months of age in Ethiopia: A multilevel analysis using 2016 Ethiopia Demographic and Health Survey. PLoS One 14, e0225639 (2019).

40. Budu, E. et al. Maternal healthcare utilsation and complete childhood vaccination in subSaharan Africa: a cross-sectional study of 29 nationally representative surveys. BMJ Open 11, e045992 (2021).

41. Ntenda, P. A. M. Factors associated with non-and under-vaccination among children aged 12-23 months in Malawi. A multinomial analysis of the population-based sample. Pediatr. Neonatol. 60, 623-633 (2019).

42. Tsawe, M. \& Susuman, A. S. Determinants of access to and use of maternal health care 
services in the Eastern Cape, South Africa: a quantitative and qualitative investigation. BMC Res. Notes 7, 1-10 (2014).

43. Harris-Fry, H. et al. Socio-economic determinants of household food security and women's dietary diversity in rural Bangladesh: a cross-sectional study. J. Heal. Popul. Nutr. 33, 2 (2015).

44. MacDonald, N. E. Vaccine hesitancy: Definition, scope and determinants. Vaccine 33, 4161-4164 (2015).

\section{Acknowledgements}

The authors are grateful to the authority of BDHS.

\section{Author contributions}

Satyajit Kundu: Conceptualization, methodology, data curation, formal analysis, writing original draft; Subarna Kundu: Conceptualization, methodology, data curation, formal analysis, writing - original draft; Abdul-Aziz Seidu: Writing - original draft, review and editing; Joshua Okyere: Writing - original draft, review and editing; Susmita Ghosh: Writing - original draft, review and editing; Ahmed Hossain: Writing - original draft, review and editing; Najim Z. Alshahrani: Writing - original draft, review and editing; Md. Hasan Al Banna: Writing - original draft, review and editing; Md. Ashfikur Rahman: Critical review and editing; Bright Opoku Ahinkorah: Writing - original draft, review and editing. All authors read and approved the final manuscript.

\section{Competing interests}

The authors have declared that no competing interests exist. 


\section{Funding}

The author(s) received no specific funding for this work.

\begin{tabular}{|c|c|c|c|c|c|c|c|}
\hline & \multicolumn{2}{|c|}{$2011(\mathrm{~N}=3098)$} & \multicolumn{2}{|r|}{$2014(\mathrm{~N}=3090)$} & \multicolumn{2}{|c|}{$2017-18(\mathrm{~N}=3313)$} & \multirow{2}{*}{$P$ value } \\
\hline & $\mathbf{n}$ & $\%(95 \% \mathrm{CI})$ & $\mathbf{n}$ & $\%(95 \% \mathrm{CI})$ & $\mathbf{n}$ & $\%(95 \% \mathrm{CI})$ & \\
\hline \multicolumn{8}{|c|}{ Vaccination card $^{\text {a }}$} \\
\hline Yes, seen & 1938 & $5.06(4.34,5.89)$ & 2175 & $69.69(68.07,71.26)$ & 2355 & $70.63(69.06,72.14)$ & \multirow{4}{*}{$<0.001$} \\
\hline Yes, Not seen & 811 & $61.29(59.56,63.00)$ & 764 & $25.87(24.38,27.42)$ & 607 & $18.71(17.43,20.07)$ & \\
\hline $\begin{array}{l}\text { No longer has } \\
\text { card }\end{array}$ & 214 & $26.00(24.49,27.58)$ & 51 & $1.45(1.09,1.93)$ & 231 & $7.12(6.30,8.04)$ & \\
\hline No card & 134 & $7.64(6.76,8.64)$ & 100 & $2.98(2.44,3.63)$ & 120 & $3.54(2.97,4.22)$ & \\
\hline \multicolumn{8}{|c|}{ Reported vaccinations from vaccination card or mother recall } \\
\hline $\mathrm{BCG}^{\mathrm{a}}$ & 3017 & $97.33(96.70,97.85)$ & 3000 & $97.70(97.12,98.17)$ & 3261 & $98.50(98.02,98.86)$ & $<0.001$ \\
\hline Polio $1^{\mathrm{b}}$ & 3024 & $97.66(97.07,98.14)$ & 2990 & $97.48(96.87,97.97)$ & 3253 & $98.31(97.81,98.69)$ & $<0.001$ \\
\hline Polio $2^{\mathrm{c}}$ & 2969 & $95.82(95.06,96.48)$ & 2940 & $96.12(95.39,96.73)$ & 3210 & $97.07(96.44,97.59)$ & 0.001 \\
\hline Polio $3^{\mathrm{c}}$ & 2887 & $93.25(92.31,94.08)$ & 2847 & $92.88(91.93,93.72)$ & 3135 & $94.83(94.03,95.53)$ & 0.001 \\
\hline \multicolumn{8}{|c|}{\begin{tabular}{|l|} 
Polio vaccination completion $(\mathrm{OPV} 1-3)^{b}$ \\
\end{tabular}} \\
\hline Full & 2886 & $93.13(92.18,93.97)$ & 2846 & $92.78(91.83,93.63)$ & 3133 & $94.65(93.84,95.37)$ & \multirow{3}{*}{$<0.001$} \\
\hline Partial & 138 & $4.53(3.85,5.33)$ & 145 & $4.74(4.05,5.53)$ & 121 & $3.70(3.11,4.39)$ & \\
\hline None & 74 & $2.34(1.86,2.93)$ & 99 & $2.48(1.99,3.08)$ & 59 & $1.65(1.27,2.14)$ & \\
\hline DTP $1^{\mathrm{b}}$ & 3017 & $97.45(96.83,97.95)$ & 2980 & $96.87(96.20,97.42)$ & 3257 & $98.41(97.93,98.79)$ & $<0.001$ \\
\hline DTP $2^{\mathrm{d}}$ & 2963 & $95.54(94.75,96.21)$ & 2963 & $95.65(94.89,96.31)$ & 3229 & $97.54(96.96,98.01)$ & $<0.001$ \\
\hline DTP $3^{\mathrm{d}}$ & 2883 & $92.97(92.01,93.81)$ & 2838 & $92.40(91.43,93.27)$ & 3175 & $96.01(95.30,96.63)$ & $<0.001$ \\
\hline \multicolumn{8}{|c|}{ DTP vaccination completion $(\text { DTP } 1-3)^{b}$} \\
\hline Full & 2882 & $92.96(92.00,93.81)$ & 2838 & $92.38(91.41,93.26)$ & 3175 & $96.01(95.30,96.63)$ & $<0.001$ \\
\hline Partial & 135 & $4.49(3.81,5.28)$ & 142 & $4.48(3.82,5.26)$ & 82 & $2.40(1.93,2.98)$ & \\
\hline None & 80 & $2.55(2.05,3.17)$ & 110 & $3.13(2.58,3.80)$ & 56 & $1.59(1.21,2.07)$ & \\
\hline Measles $^{\mathrm{e}}$ & 2747 & $88.66(87.49,89.73)$ & 2668 & $87.22(86.01,88.33)$ & 3020 & $91.33(90.33,92.24)$ & $<0.001$ \\
\hline \multicolumn{8}{|c|}{ Vaccination status $f$} \\
\hline Full & 2694 & $86.17(85.46,87.86)$ & 2611 & $85.13(83.86,86.33)$ & 2954 & $89.23(88.13,90.23)$ & \multirow{3}{*}{$<0.001$} \\
\hline Partial & 336 & $11.13(10.07,12.29)$ & 399 & $12.89(11.78,14.10)$ & 311 & $9.42(8.48,10.46)$ & \\
\hline None & 68 & $2.16(1.70,2.74)$ & 80 & $1.97(1.54,2.52)$ & 48 & $1.35(1.01,1.80)$ & \\
\hline \multicolumn{8}{|c|}{$\begin{array}{l}\text { a } 2011 n=3097 ; 2014 n=3090 ; 2017-18 n=3313 \\
\text { b } 2011 n=3098 ; 2014 n=3090 ; 2017-18 n=3313 \\
\text { c } 2011 n=3095 ; 2014 n=3087 ; 2017-18 n=3311 \\
\text { d } 2011 n=3097 ; 2014 n=3089 ; 2017-18 n=3313 \\
\text { e } 2011 n=3095 ; 2014 n=3086 ; 2017-18 n=3313 \\
{ }^{f} 2011 n=3098 ; 2014 n=3090 ; 2017-18 n=3313\end{array}$} \\
\hline
\end{tabular}




\begin{tabular}{|c|c|c|c|c|c|c|c|c|c|c|c|c|}
\hline \multirow{3}{*}{ Variables } & \multicolumn{12}{|c|}{ Fully vaccinated } \\
\hline & \multicolumn{3}{|c|}{$2011(\mathrm{~N}=2694)$} & \multicolumn{3}{|c|}{$2014(N=2611)$} & \multicolumn{3}{|c|}{ 2017-18 $(\mathrm{N}=2954)$} & \multicolumn{3}{|c|}{ Pooled $(\mathrm{N}=8259)$} \\
\hline & $\mathbf{n}$ & $\%(95 \% \mathrm{CI})$ & $\mathbf{p}$ & $\mathbf{n}$ & $\%(95 \% \mathrm{CI})$ & $\mathbf{p}$ & $n$ & $\%(95 \% \mathrm{CI})$ & $\mathbf{p}$ & $\mathbf{n}$ & $\%(95 \% \mathrm{CI})$ & $\mathbf{p}$ \\
\hline \multicolumn{13}{|c|}{ Individual and household level variables } \\
\hline \multicolumn{13}{|l|}{ Sex of child } \\
\hline Female & 1339 & $84.97(83.11,86.65)$ & \multirow{2}{*}{0.005} & 1289 & $86.05(84.22,87.70)$ & \multirow{2}{*}{0.426} & 1414 & $88.41(86.82,89.83)$ & \multirow{2}{*}{0.560} & 4217 & $87.08(86.09,88.01)$ & \multirow{2}{*}{0.392} \\
\hline Male & 1355 & $88.51(86.80,90.02)$ & & 1322 & $84.30(82.47,85.97)$ & & 1540 & $90.11(88.55,91.47)$ & & 4042 & $88.05(86.09,88.01)$ & \\
\hline \multicolumn{13}{|l|}{ Current age of child } \\
\hline $12-23$ months & 1328 & $85.94(84.11,87.58)$ & \multirow{2}{*}{0.168} & 1281 & $83.79(81.92,85.50)$ & \multirow{2}{*}{0.002} & 1463 & $88.27(86.64,89.72)$ & \multirow{2}{*}{0.044} & 4072 & $86.02(85.01,86.97)$ & \multirow{2}{*}{$<0.001$} \\
\hline $24-35$ months & 1366 & $87.48(85.73,89.04)$ & & 1330 & $86.54(84.75,88.14)$ & & 1491 & $90.18(88.67,91.52)$ & & 4187 & $88.12(87.17,89.01)$ & \\
\hline \multicolumn{13}{|l|}{ Maternal age } \\
\hline$<25$ years & 1436 & $87.56(85.88,89.07)$ & \multirow{3}{*}{0.068} & 1357 & $85.34(83.54,86.97)$ & \multirow{3}{*}{0.307} & 1464 & $88.07(86.45,89.52)$ & \multirow{3}{*}{0.102} & 4257 & $87.01(86.05,87.91)$ & \\
\hline $25-34$ years & 1079 & $86.30(84.28,88.10)$ & & 1082 & $84.90(82.88,86.73)$ & & 1293 & $90.47(88.84,91.89)$ & & 3454 & $87.32(86.25 .88 .32)$ & 0.061 \\
\hline$>34$ years & 179 & $82.08(76.04,86.86)$ & & 172 & $85.04(79.73,89.14)$ & & 197 & $90.21(85.43,93.53)$ & & 548 & $85.87(82.93,88.38)$ & \\
\hline Maternal education & & & & & & & & & & & & \\
\hline No education & 404 & $75.94(72.24,79.29)$ & & 295 & 72.24(67.97,76.15) & & 170 & $78.60(72.69,83.53)$ & & 869 & $75.04(72.56,77.37)$ & \\
\hline Primary & 764 & $83.41(80.90,85.65)$ & ( & 688 & $79.89(77.13,82.39)$ & ( & 778 & $84.71(82.27,86.87)$ & ( & 2230 & $82.72(81.27,84.08)$ & 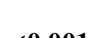 \\
\hline Secondary & 1235 & $91.69(90.07,93.06)$ & $<0.001$ & 1303 & $90.18(88.59,91.58)$ & $<0.001$ & 1451 & $91.36(89.89,92.63)$ & $<0.001$ & 3989 & $91.05(90.18,91.85)$ & $<0.001$ \\
\hline Higher & 291 & $97.05(94.07,98.56)$ & & 325 & $94.50(91.33,96.56)$ & & 555 & $94.73(92.57,96.29)$ & & 1171 & $95.19(93.77,96.29)$ & \\
\hline Wealth index & & & & & & & & & & & & \\
\hline Poor & 1031 & $82.43(80.31,84.37)$ & & 964 & $77.30(74.94,79.49)$ & & 1210 & $87.38(85.52,89.03)$ & & 3205 & $82.46(81.26,83.60)$ & \\
\hline Middle & 506 & $87.92(85.07,90.29)$ & $<0.001$ & 508 & $88.70(85.87 .91 .02)$ & $<0.001$ & 520 & $90.14(87.57,92.23)$ & 0.001 & 1534 & $88.95(87.42,90.30)$ & $<0.001$ \\
\hline Rich & 1157 & $91.16(89.36,92.68)$ & & 1139 & $91.39(89.74,92.80)$ & & 1224 & $90.69(89.02,92.14)$ & & 3520 & $91.07(90.12,91.94)$ & \\
\hline Media access & & & & & & & & & & & & \\
\hline Never/less than once & 1256 & $83.70(81.79,85.45)$ & & 1176 & $79.25(77.16,81.20)$ & & 1306 & $87.43(85.63,89.03)$ & 0003 & 3738 & $83.39(82.28,84.44)$ & \\
\hline At least once a week & 1438 & $89.82(88.19,91.24)$ & $<0.001$ & 1435 & $90.69(89.18,92.00)$ & $<0.001$ & 1648 & $90.63(89.23,91.86)$ & 0.002 & 4521 & $90.41(89.56,91.19)$ & $<0.001$ \\
\hline Place of delivery & & & & & & & & & & & & \\
\hline Home & 1835 & $84.15(82.58,85.60)$ & (0001 & 1568 & $81.73(79.97,83.36)$ & & 1452 & $87.02(85.35,88.53)$ & 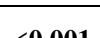 & 4855 & $84.16(83.22,85.07)$ & \\
\hline Health facility & 859 & $93.55(91.67,95.03)$ & $<0.001$ & 1043 & $90.85(89.08,92.37)$ & $<0.001$ & 1502 & $91.54(90.09,92.80)$ & $<0.001$ & 3404 & $91.78(90.84,92.63)$ & $<0.001$ \\
\hline ANC visit & & & & & & & & & & & & \\
\hline No visit & 710 & $79.12(76.39,81.61)$ & & 484 & $73.93(70.50,77.09)$ & & 196 & $82.12(76.78,86.46)$ & & 1390 & $77.61(75.65,79.45)$ & \\
\hline 1 to 3 visits & 1064 & $90.16(88.36,91.71)$ & 5001 & 1139 & $86.06(84.13,87.79)$ & & 1148 & $87.33(85.48,88.98)$ & $<0001$ & 3351 & $87.76(86.71,88.74)$ & \\
\hline 4 or more visits & 737 & $92.41(90.20,94.16)$ & $<0.001$ & 842 & $92.55(90.68,94.07)$ & $<0.001$ & 1434 & $92.38(90.93,93.61)$ & $<0.001$ & 3013 & $92.44(91.46,93.31)$ & $<0.001$ \\
\hline Others" ${ }^{\#}$ & 226 & $81.90(76.43,86.33)$ & & 146 & $82.34(76.49,86.99)$ & & 176 & $87.08(81.70 .91 .05)$ & & 505 & $83.67(80.60,86.34)$ & \\
\hline Number of children & & & & & & & & & & & & \\
\hline Single & 1018 & $89.75(87.82,91.41)$ & & 1065 & $86.96(84.95,88.74)$ & & 1147 & $90.44(88.72,91.92)$ & & 3230 & $89.07(88.01,90.04)$ & \\
\hline Two children & 867 & $87.01(84.80,88.94)$ & $<0.001$ & 860 & $87.90(85.79,89.73)$ & $<0.001$ & 1030 & $89.16(87.24,90.82)$ & 0.118 & 2757 & $88.08(86.91,89.15)$ & $<0.001$ \\
\hline More than two & 809 & $82.93(80.43,85.17)$ & & 686 & $79.53(76.79,82.02)$ & & 777 & $87.52(85.17,89.54)$ & & 2272 & $83.26(81.82,84.60)$ & \\
\hline Community level vari & & & & & & & & & & & & \\
\hline Divisions & & & & & & & & & & & & \\
\hline Barisal & 304 & $86.40(80.39,90.78)$ & & 300 & $83.81(77.71 .88 .50)$ & & 289 & $87.19(81.42 .91 .35)$ & ( & 893 & $85.78(82.54,88.50)$ & \\
\hline Chittagong & 529 & $83.12(80.20,85.68)$ & $<0.001$ & 524 & $84.45(81.60,86.93)$ & $<0.001$ & 501 & $88.46(85.93,90.58)$ & 0.005 & 1554 & $85.36(83.80,86.78)$ & $<0.001$ \\
\hline Dhaka & 439 & $86.39(84.06,88.42)$ & & 484 & $88.18(86.16,89.93)$ & & 786 & $88.50(86.51,90.23)$ & & 1709 & $87.76(86.58,88.85)$ & \\
\hline
\end{tabular}




\begin{tabular}{|c|c|c|c|c|c|c|c|c|c|c|c|c|}
\hline Khulna & 329 & $91.70(87.91,94.38)$ & & 308 & $87.50(82.85,91.02)$ & & 303 & $92.10(88.38,94.70)$ & & 940 & $90.55(88.37,92.36)$ & \\
\hline Rajshahi & 351 & $90.19(86.87,92.75)$ & & 330 & $86.52(82.29,89.86)$ & & 323 & $91.27(88.06,93.68)$ & & 1004 & $89.54(87.59,91.20)$ & \\
\hline Rangpur & 342 & $91.31(87.64,93.97)$ & & 350 & $93.37(89.96,95.68)$ & & 340 & $92.20(88.92,94.57)$ & & 1032 & $92.28(90.43,93.80)$ & \\
\hline Sylhet & 400 & $81.02(75.47,85.56)$ & & 315 & $63.81(58.15,69.11)$ & & 412 & $85.72(81.05,89.40)$ & & 1127 & $76.30(73.22,79.12)$ & \\
\hline \multicolumn{13}{|c|}{ Place of residence } \\
\hline Urban & 902 & $88.78(86.27,90.88)$ & \multirow{2}{*}{0.001} & 845 & $87.77(85.35,89.84)$ & \multirow{2}{*}{0.071} & 1020 & $88.51(86.27,90.44)$ & \multirow{2}{*}{0.920} & 2767 & $88.34(87.01,89.55)$ & \multirow{2}{*}{0.002} \\
\hline Rural & 1792 & $86.07(84.61,87.41)$ & & 1766 & $84.22(82.70,85.63)$ & & 1934 & $89.49(88.21,90.64)$ & & 5492 & $86.63(85.82,87.39)$ & \\
\hline \multicolumn{13}{|c|}{ Community literacy level of women } \\
\hline High & 1409 & $90.82(89.23,92.19)$ & \multirow{2}{*}{$<0.001$} & 1218 & $88.72(87.11,90.15)$ & \multirow[b]{2}{*}{$<0.001$} & 1609 & $92.01(90.64,93.19)$ & \multirow{2}{*}{$<0.001$} & 4455 & $90.89(9005,91.66)$ & \multirow{2}{*}{$<0.001$} \\
\hline Low & 1285 & $82.98(81.07,84.73)$ & & 1393 & $81.20(79.16,83.09)$ & & 1345 & $86.24(84.47,87.83)$ & & 3804 & $83.14(82.05,84.18)$ & \\
\hline \multicolumn{13}{|c|}{ Community wealth index level } \\
\hline High & 1376 & $85.84(84.00,87.49)$ & \multirow{2}{*}{0.017} & 1270 & $80.63(78.64,82.47)$ & \multirow{2}{*}{$<0.001$} & 1528 & $88.80(87.24,90.19)$ & \multirow{2}{*}{0.034} & 4014 & $85.16(84.14,86.12)$ & \multirow{2}{*}{$<0.001$} \\
\hline Low & 1318 & $87.57(85.83,89.13)$ & & 1341 & $89.91(88.30,91.31)$ & & 1426 & $89.70(88.11,91.10)$ & & 4245 & $89.07(88.14,89.93)$ & \\
\hline \multicolumn{13}{|c|}{ Community media exposure } \\
\hline High & 1455 & $89.10(87.51,90.51)$ & \multirow{2}{*}{$<0.001$} & 1174 & $89.78(88.24,91.14)$ & \multirow{2}{*}{$<0.001$} & 1508 & $89.92(88.41,91.25)$ & \multirow{2}{*}{0.020} & 4356 & $89.26(88.37,90.08)$ & \multirow[b]{2}{*}{$<0.001$} \\
\hline Low & 1239 & $83.92(81.92,85.74)$ & & 1437 & $80.00(77.91,81.94)$ & & 1446 & $88.48(86.83,89.95)$ & & 3903 & $84.62(83.55,85.64)$ & \\
\hline Total & 2694 & $86.71(85.46,87.86)$ & & 2611 & $85.13(83.86,86.33)$ & & 2954 & $89.23(88.13,90.23)$ & & 8259 & $87.06(86.38,87.72)$ & \\
\hline
\end{tabular}




\begin{tabular}{|c|c|c|c|c|c|c|c|c|}
\hline \multirow[t]{2}{*}{ Variables } & \multicolumn{2}{|l|}{2011} & \multicolumn{2}{|l|}{2014} & \multicolumn{2}{|l|}{ 2017-18 } & \multicolumn{2}{|l|}{ Pooled } \\
\hline & $\operatorname{AOR}(95 \% \mathrm{CI})$ & p value & $\operatorname{AOR}(95 \% \mathrm{CI})$ & $p$ value & $\operatorname{AOR}(95 \% \mathrm{CI})$ & p value & AOR $(95 \% \mathrm{CI})$ & $\begin{array}{c}\mathbf{p} \\
\text { value }\end{array}$ \\
\hline \multicolumn{9}{|c|}{ Individual and household level variables } \\
\hline \multicolumn{9}{|l|}{ Sex of child } \\
\hline Male & Ref & & Ref & & Ref & & Ref & \\
\hline Female & $0.69(0.55,0.88)$ & 0.002 & $1.00(0.80,1.26)$ & 0.990 & $1.06(0.83,1.35)$ & 0.650 & $0.93(0.81,1.05)$ & 0.241 \\
\hline \multicolumn{9}{|l|}{ Current age of child } \\
\hline $12-23$ months & Ref & & Ref & & Ref & & Ref & \\
\hline $24-35$ months & $1.31(1.03,1.67)$ & 0.029 & $1.63(1.29,2.06)$ & $<0.001$ & $1.31(1.03,1.69)$ & 0.031 & $1.40(1.22,1.59)$ & $<0.001$ \\
\hline \multicolumn{9}{|l|}{ Maternal age } \\
\hline$<25$ years & Ref & & Ref & & Ref & & Ref & \\
\hline 25-34 years & $0.91(0.66,1.25)$ & 0.553 & $1.58(1.15,2.17)$ & 0.004 & $1.51(1.09,2.08)$ & 0.014 & $1.30(1.09,1.54)$ & 0.004 \\
\hline$>34$ years & $0.80(0.48,1.35)$ & 0.404 & $2.06(1.21,3.51)$ & 0.008 & $1.78(0.98,3.22)$ & 0.059 & $1.39(1.03,1.87)$ & 0.031 \\
\hline \multicolumn{9}{|l|}{ Maternal education } \\
\hline No education & Ref & & Ref & & Ref & & Ref & \\
\hline Primary & $1.45(1.06,1.99)$ & 0.020 & $1.54(1.11,2.13)$ & 0.010 & $1.44(0.94,2.22)$ & 0.094 & $1.53(1.27,1.84)$ & $<0.001$ \\
\hline Secondary & $2.31(1.59,3.34)$ & $<0.001$ & $2.11(1.47,3.03)$ & $<0.001$ & $2.36(1.49,3.74)$ & $<0.001$ & $2.39(1.94,2.94)$ & $<0.001$ \\
\hline Higher & $7.28(3.05,17.39)$ & $<<0.001$ & $4.29(2.22,8.32)$ & $<0.001$ & $3.79(2.05,7.02)$ & $<0.001$ & $4.51(3.15,6.45)$ & $<0.001$ \\
\hline \multicolumn{9}{|l|}{ Wealth index } \\
\hline Poor & Ref & & Ref & & Ref & & Ref & \\
\hline Middle & $1.14(0.81,1.61)$ & 0.437 & $1.10(0.79,1.54)$ & 0.574 & $1.09(0.74,1.59)$ & 0.667 & $1.11(0.92,1.35)$ & 0.266 \\
\hline Rich & $1.46(1.01,2.12)$ & 0.045 & $1.45(1.01,2.12)$ & 0.049 & $1.00(0.68,1.47)$ & 0.998 & $1.27(1.04,1.55)$ & 0.020 \\
\hline \multicolumn{9}{|l|}{ Media access } \\
\hline Never/ less than once & Ref & & Ref & & Ref & & Ref & \\
\hline At least once a week & $0.86(0.64,1.15)$ & 0.309 & $1.35(0.99,1.82)$ & 0.053 & $0.90(0.67,1.22)$ & 0.508 & $1.07(0.91,1.26)$ & 0.412 \\
\hline \multicolumn{9}{|l|}{ Place of delivery } \\
\hline Home & Ref & & Ref & & Ref & & Ref & \\
\hline Health facility & $1.44(1.01,2.04)$ & 0.042 & $0.90(0.68,1.20)$ & 0.480 & $1.28(0.96,1.70)$ & 0.087 & $1.15(0.97,1.35)$ & 0.103 \\
\hline \multicolumn{9}{|l|}{ ANC visit } \\
\hline No visit & Ref & & Ref & & Ref & & Ref & \\
\hline 1 to 3 visits & $1.42(0.96,2.10)$ & 0.079 & $1.49(1.12,1.99)$ & 0.007 & $1.13(0.74,1.73)$ & 0.580 & $1.41(1.19 .1 .66)$ & $<0.001$ \\
\hline 4 or more visits & $1.52(1.14,2.03)$ & 0.005 & $1.73(1.19,2.50)$ & 0.004 & $1.63(1.01,2.61)$ & 0.044 & $1.77(1.44,2.17)$ & $<0.001$ \\
\hline Others $^{\#}$ & $0.90(0.57,1.40)$ & 0.635 & $1.26(0.77,2.07)$ & 0.348 & $1.22(0.66,2.26)$ & 0.521 & $1.09(0.83,1.43)$ & 0.533 \\
\hline \multicolumn{9}{|l|}{ Number of children } \\
\hline Single & Ref & & Ref & & Ref & & Ref & \\
\hline Two & $0.89(0.64,1.23)$ & 0.465 & $1.02(0.75,1.39)$ & 0.900 & $0.94(0.68,1.30)$ & 0.702 & $0.97(0.81,1.16)$ & 0.730 \\
\hline More than two & $1.13(0.76,1.69)$ & 0.553 & $0.63(0.43,0.93)$ & 0.021 & $0.89(0.58,1.38)$ & 0.612 & $0.86(0.69,1.08)$ & 0.195 \\
\hline Community level varia & & & & & & & & \\
\hline Divisions & & & & & & & & \\
\hline Barisal & Ref & & Ref & & Ref & & Ref & \\
\hline Chittagong & $0.79(0.47,1.32)$ & 0.364 & $1.01(0.60,1.68)$ & 0.967 & $0.99(0.56,1.75)$ & 0.983 & $0.91(0.66,1.25)$ & 0.555 \\
\hline Dhaka & $1.03(0.60,1.79)$ & 0.902 & $1.05(0.62,1.79)$ & 0.845 & $1.17(0.69,1.98)$ & 0.562 & $1.21(0.90,1.62)$ & 0.209 \\
\hline Khulna & $1.35(0.72,2.54)$ & 0.353 & $0.74(0.42,1.30)$ & 0.292 & $1.32(0.67,2.59)$ & 0.424 & $1.04(0.73,1.49)$ & 0.819 \\
\hline Rajshahi & $1.72(0.94,3.15)$ & 0.078 & $1.33(0.76,2.35)$ & 0.317 & $1.43(0.75,2.70)$ & 0.276 & $1.62(1.13,2.31)$ & 0.008 \\
\hline Rangpur & $1.51(0.83,2.73)$ & 0.177 & $2.10(1.16,3.81)$ & 0.015 & $1.65(0.86,3.15)$ & $\mathbf{0 . 1 3 3}$ & $1.72(1.32,2.79)$ & 0.041 \\
\hline Sylhet & $0.85(0.50,1.46)$ & 0.556 & $0.37(0.23,0.61)$ & $<0.001$ & $0.98(0.55,1.73)$ & 0.938 & $0.97(0.65,1.45)$ & 0.883 \\
\hline Place of residence & & & & & & & & \\
\hline Urban & $1.10(0.77,1.56)$ & 0.596 & $1.48(1.06,2.06)$ & 0.021 & $1.24(0.88,1.74)$ & 0.217 & $1.25(1.05,1.49)$ & 0.012 \\
\hline Rural & Ref & & Ref & & Ref & & Ref & \\
\hline Community literacy l & el of women & & & & & & & \\
\hline High & $1.46(1.04,2.06)$ & 0.030 & $1.19(0.86,1.64)$ & 0.293 & $1.46(1.03,2.05)$ & 0.032 & $1.36(1.12,1.65)$ & 0.002 \\
\hline Low & Ref & & Ref & & Ref & & Ref & \\
\hline Community wealth in & $\mathrm{x}$ level & & & & & & & \\
\hline High & $1.21(0.85,1.73)$ & 0.294 & $0.85(0.60,1.22)$ & 0.390 & $0.96(0.65,1.42)$ & 0.845 & $1.03(0.84,1.27)$ & 0.747 \\
\hline Low & Ref & & Ref & & Ref & & Ref & \\
\hline Commt & sure & & & & & & & \\
\hline High & $1.19(0.83,1.70)$ & 0.353 & $1.00(0.70,1.42)$ & 0.992 & $0.96(0.66,1.40)$ & 0.826 & $1.02(0.83,1.26)$ & 0.842 \\
\hline Low & Ref & & Ref & & Ref & & Ref & \\
\hline Rando & & & & & & & & \\
\hline Cluster effects $(95 \% \mathrm{CI})$ & $0.78(0.60,1.01)$ & $<0.001$ & $0.79(0.62,0.99)$ & $<0.001$ & $0.88(0.69,1.12)$ & $<0.001$ & $0.59(0.50,0.70)$ & $<0.001$ \\
\hline
\end{tabular}




\begin{tabular}{|c|c|c|c|c|c|}
\hline ICC (95\% CI) & $0.16(0.09,0.23)$ & $0.16(0.11,0.23)$ & $0.19(0.13,0.28)$ & $0.10(0.07,0.13)$ & \\
\hline AIC & 2198.66 & 2364.61 & 2165.996 & 6794.70 & \\
\hline \multicolumn{6}{|c|}{$\begin{array}{l}\text { \# Others included missing values and don't know, } \\
\text { The bolded p values indicate the statistical significance, AOR=Adjusted Odds ratio, CI=Confidence Interval. } \\
\text { ICC= Intraclass Correlation Coefficient; AIC= Akaike's Information Criterion. } \\
\text { a Significance of cluster random effects is assessed using log-likelihood ratio test (LR test vs logistic model). }\end{array}$} \\
\hline
\end{tabular}


Figures

\begin{tabular}{|c|c|c|c|c|c|c|}
\hline Vaccine & Disease & At birth & 6 weeks & 10 weeks & 14 weeks & 9 months \\
\hline BCG & Tuberculosis & $\sqrt{ }$ & & & & \\
\hline OPV & Polio & & $\sqrt{ }$ & $\sqrt{ }$ & $\sqrt{ }$ & \\
\hline DTP & $\begin{array}{l}\text { Diphtheria, Tetanus, } \\
\text { and Pertussis }\end{array}$ & & $\sqrt{ }$ & $\sqrt{ }$ & $\sqrt{ }$ & \\
\hline Measles & Measles & & & & & $\sqrt{ }$ \\
\hline
\end{tabular}

Figure 1

Basic vaccination administration schedule for children under 12 months in Bangladesh. 


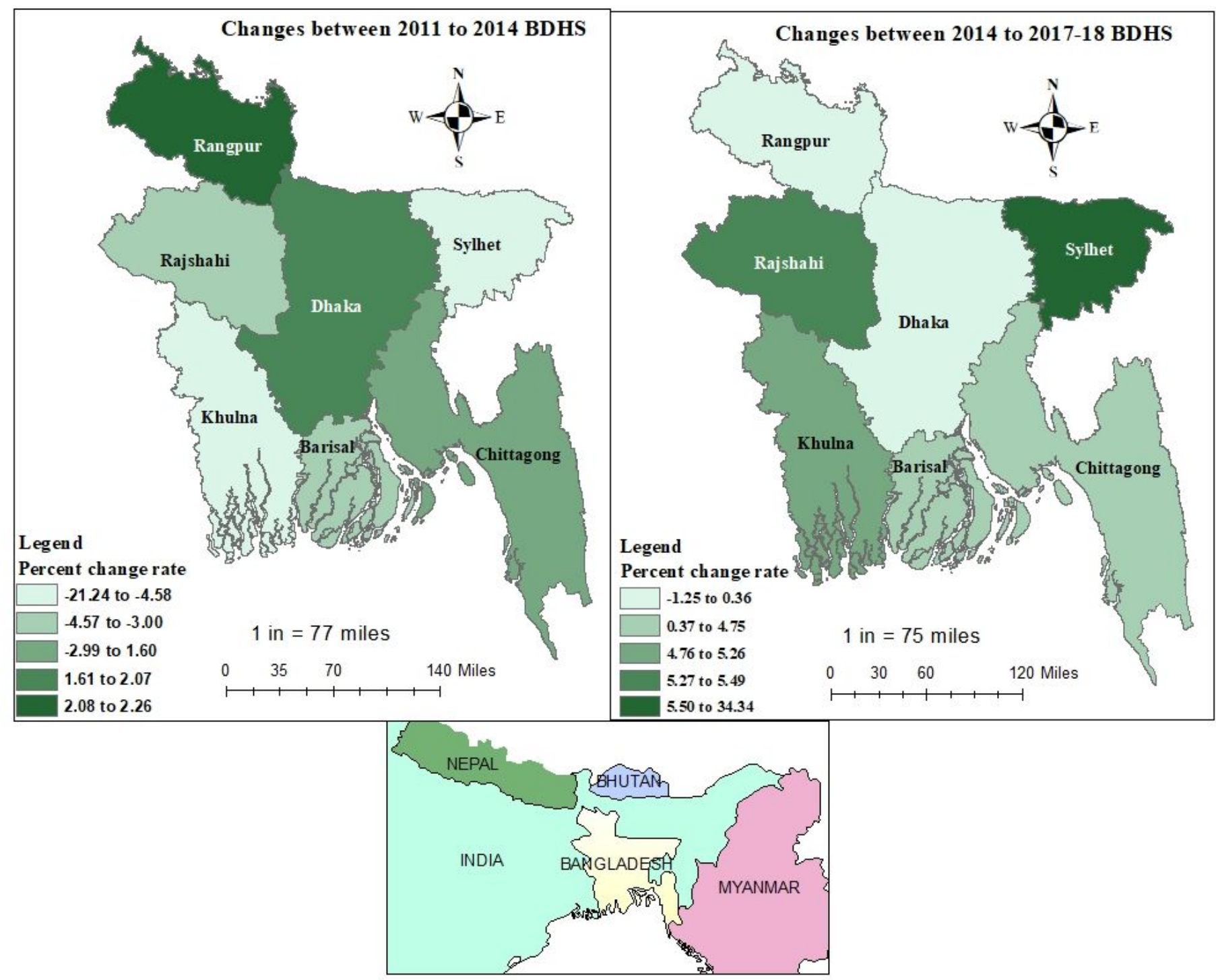

Figure 2

Spatial distribution of change rate in vaccination status among children aged 12-35 months old from BDHS 2011 to 2014 and BDHS 2014 to 2017-18. 